\section{THE TELECOMMUNICATIONS RESEARCH ESTABLISHMENT, MALVERN}

$\mathrm{A}^{\mathrm{N}}$ $\mathrm{N}$ exhibition of the work carried out at the Telecommunications Research Establishment of the Ministry of Supply was held at Great Malvern during May 25-27. This was the first occasion on which this well-known establishment has been open to the public. The Telecommunications Research Establishment, or T.R.E. as it is generally called, became famous during the war years on account of the pioneering research on radar carried out there. It developed from the small research group at Bawdsey originally under Sir Robert Watson-Watt to become one of the largest Government research establishments and attracted many of the leading scientific workers in Britain.

During the War, T.R.E. was responsible for research and development of most of the radar equipment for the Royal Air Force. The story of this work is now very well known. At the close of hostilities many of the leading scientific workers at T.R.E., as was indeed expected, returned to academic life. A considerable number of war-time recruits, however, stayed on in the Government Scientific Service and have carried on the tradition of T.R.E. After the War the activities of the Establishment were broadened in scope and a considerable part of the effort on military problems was turned to civil problems and to fundamental research.

The Establishment is now divided into three Departments-Physics, Radar and Engineering. The Physics Department is mainly concerned with fundamental research on the properties of extremely short radio waves, on infra-red radiation and on various fundamental problems in electronies. It includes a theoretical physics group. Some research on supersonics is also being carried out and the application of radar techniques to various problems in meteorology is being studied. A considerable part of the work of the Physics Department is supported and financed by the Department of Scientific and Industrial Research. The Radar Department is responsible for research and development of radar equipments for the Royal Air Force, the Naval Air Arm and the Ministry of Civil Aviation. Examples of projects on which research is taking place are the location by radar of bombing targets, the location and attack of enemy aircraft, radio navigational aids and the use of radar for the control of air traffic. The Engineering Department is responsible for the design and construction of apparatus to the requirements of the scientific staff and for investigating new materials, finishes and component techniques to meet the wide variations of temperature and humidity encountered at high altitudes and in various parts of the world. It includes the Engineering Unit, which is one of the largest and best equipped workshops in Britain.

The Ministry of Supply School of Electronics is situated within T.R.E. Technical instruction is provided in the School for craft and engineering apprentices. Radar courses are arranged for all classes of new entrants, and advanced refresher courses for internal staff. A department of the Atomic Energy Research Establishment is also at present situated at T.R.E. It is concerned with the development of high-energy particle accelerators for nuclear physics and medical research work, and with electronic instrumentation for measuring all forms of radiation in the field of nuclear physics.

The exhibition was opened on May 25 by the Right Hon. G. R. Strauss, Minister of Supply. On that day leading representatives of the Fighting Services, the Civil Service, universities and industry, together with representatives of the Press, were shown a selection of the work of the Establishment. On May 26, about 1,400, representing other Government establishments, industry, and the universities and technical colleges, attended the exhibition. On May 27, senior science scholars from local schools and members of the public from Malvern and nearby towns attended the exhibition. This was made an occasion for the formal opening of the Ministry of Supply School of Electronics by Mr. J. Freeman, M.P., Parliamentary Secretary to the Ministry of Supply.

It was clearly possible to show only a selection of the work of T.R.E. 'This selection was representative of that carried out in all departments, and items were chosen which were free from any security restrictions. A few of the more interesting exhibits are described below.

\section{Application of Radar to Meteorology}

A number of exhibits illustrated the application of radar techniques to meteorology. Photographs of rain clouds and of the radar echoes obtained from them were shown. A light-weight microwave radar equipment, for installation in large civil aircraft, was demonstrated. This equipment is to enable aircraft to avoid dangerous clouds, particularly those of the cumulo-nimbus type. Fortunately this type of cloud gives an excellent radar echo at a wavelength of $3 \mathrm{~cm}$. at which the equipment operates. An interesting problem in the study of rain is the determination of the size of raindrops, particularly immediately below precipitating clouds. A method of making this kind of measurement by use of a specially designed microphone in an aircraft was exhibited. An interesting application of pulsed light to the measurement of the height of the cloud base was also shown. Another application of radar techniques is the radar sonde. Radar is used to locate the position of the sonde carried on a balloon. Thus observations of wind speed can be made from a single station. Meteorological information as determined by the instruments carried in the sonde is transmitted to the ground by the train of radar pulses.

\section{New Developments in Microwave Technique}

A number of exhibits illustrated the development of microwave technique for wave-lengths near $3-10 \mathrm{~cm}$. These included demonstrations showing the improvement of receiver performance and the development of very high power transmitters, both aimed at increasing the range of detection by radar equipment. The development of techniques to enable even shorter wave-lengths to be used was illustrated. One exhibit, for example, showed the method of construction of valves for wave-lengths of the order of 8 millimetres and of the associated microwave components.

\section{D.C. Amplifier using Magnetic Modulator}

The amplification of small direct currents is much more difficult than that of small alternating currents, 
and the technique generally adopted is to turn the direct current into alternating current before amplification. A new type of amplifier which uses a magnetic modulator to effect this conversion was exhibited. The apparatus is particularly suitable for amplification of small signals from low-impedance sources such as thermocouples.

\section{Electronic Digital Computing Techniques}

An electronic memory' for a computing system was demonstrated. This is based on the storage of an electrostatic charge distribution produced on the fluorescent screen of a cathode ray tube by a bombarding beam of electrons. Many binary numbers of 32 digits can be simultaneously remembered by this system, which has been developed in association with Prof. F. C. Williams of the University of Manchester. A binary number arithmetical unit was also shown which will add together two $\mathbf{3 2}$ digit binary numbers in a time of the order of 350 microseconds.

\section{Infra-red Research}

A number of exhibits illustrating the use of infrared radiation for the detection of temperature differences were shown. For radiations in the range of 1-3 microns the very rapid response of the lead sulphide type of cell makes it useful for the detection of temperature changes which are very short-lived. One exhibit illustrated its use for the detection of hot spots caused by friction. These may last only a small fraction of a millisecond but are clearly shown by the lead sulphide detector. The use of an electronic A.C. amplifier operating at a frequency of 5 cycles per second in conjunction with a thermocouple of special design was shown as an example of an extremely sensitive detector of radiation arising from bodies having only a small difference in temperature from their surroundings. The amplifier is thought to be the best of its type designed so far and is capable of showing the Johnson noise from a 1-ohm resistance in a band-width of 1 leycle per second.

\section{Automatic Recording Spectrophotometer}

An automatic recording spectrophotometer which has been designed for the Medical Research Council was exhibited. This instrument enables an ultraviolet absorption spectrum of a substance in liquid solution to be obtained as a trace on photographic paper. The wave-length scale and absorption are simultaneously recorded on the photographic paper so that on development complete information is obtained without further calibration. This enables specimens to be examined with extreme ease and rapidity. The instrument provides an excellent example of the use of electronic servo mechanisms.

\section{Automatic Following Telescope}

A working model of an astronomical telescope locked by electronic means to a star at which it is pointed was shown. The telescope contains a device capable of determining the position of a star in its field of view, and any deviation of the star from the centre of the optical system produces correcting signals which drive the telescope to keep the star centrally aligned.

\section{Air Traffic Control}

By means of radar equipments sited at Malvern and near London airport a display showing the position of air traffic in the southern half of England was shown. The exhibit illustrated the control of aircraft arriving at a busy airport using radar information.

\section{Airborne Radar}

A number of exhibits showed the latest improvements in a number of radar navigational aids developed during the War. Another exhibit showed the radar equipment used in a modern bomber. This included the $H 2 S$ equipment for obtaining a radar map of the ground over which the aircraft is flying, and the automatic gun-laying equipment used in the rear turret. The latter equipment enables a rear gunner to open fire at an approaching fighter which he cannot see visually. Some recent developments in the radar equipment for night fighters were also shown. The equipment enables the fighter to locate and follow an enemy bomber in the dark and to mancuvre into such a position that the pilot can see the enemy aireraft and attack it A device was also shown which enables the radar information to be projected on to the pilot's windscreen so that he can at the same time view it and look out for the enemy aircraft.

\section{Suppressed Aerials}

As aircraft speeds increase, the drag of external aerials becomes more and more intolerable, and as sonic speeds are approached it is impossible to have external ayrials at all. Methods of suppressing aerials by use of plastic fittings to the aircraft and by the use of slot asrials were illustrated. These methods represent considerable advances in aircraft aorial technique.

\section{Ultrasonic Research}

A number of applications of ultrasonics were illustrated, such as the measurement of ultrasonic absorption in metals as a means of determining the grain size, and the detection of obstacles and flaws by means of ultrasonic technique. The methods used were based on radar principles and employed pulses of ultrasonic energy.

\section{Electronics for Atomic Energy}

The exhibitions of the Atomic Energy Electronics Group included an electron accelerator operating on the synchrotron principle and producing electrons having an energy of 15 million electron volts. Various instruments were also demonstrated which are used for the measurement of radiations met with in nuclear physics research. In particular, a number of instruments called 'health monitors' were shown which are used to ensure that workers are not being exposed to an amount of radiation sufficient to cause appreciable bodily harm.

The Ministry of Supply School of Electronics gave a display of equipment illustrating the historical development of microwave technique. An exhibition arranged by the Engineering Department illustrated the development of new types of radio components designed to withstand extreme climatic conditions and also showed some of the latest types of miniature radio components which have been developed with the view of reducing the size and weight of airborne radio equipment. In addition, a number of films, including "RDF to Radar", were shown. 